\title{
Estimulantes alimentares para larvas de pacu
}

\section{Marcelo Borges Tesser ${ }^{1}$, Maria Célia Portella ${ }^{2}$}

\author{
1 Universidade Federal do Rio Grande, Instituto de Oceanografia, Laboratório de Nutrição de Organismos Aquáticos, Caixa Postal 474, \\ Rio Grande - RS, 96201-900. \\ 2 Centro de Aquicultura da Unesp - Jaboticabal. Via de Acesso Prof. Paulo Donato Castellane, s/n, Jaboticabal - SP, $14884-900$.
}

RESUMO - Este estudo foi realizado com o objetivo de investigar o efeito estimulante de cinco aminoácidos (alanina, arginina, glicina, histidina e lisina) da betaína e de suas misturas sobre a taxa de ingestão de dieta microencapsulada durante o desenvolvimento larval de pacu Piaractus mesopotamicus. Os resultados da análise estatística comprovaram a existência de efeito significativo, tanto da idade das larvas como dos aminoácidos, sobre a taxa de ingestão. No entanto, não houve significância estatística para a interação dos dois fatores. A glicina, a lisina e a betaína foram considerados bons estimuladores do comportamento alimentar de larvas de pacu.

Palavras-chave: aminoácidos, estimulantes, Piaractus mesopotamicus, taxa de ingestão

\section{Feeding stimulants for pacu lavae}

\begin{abstract}
The present study aimed to investigate the stimulant effect of five amino acids (alanine, arginine, glycine, histidine and lysine) from betaine and their mixtures on the ingestion rates of formulated diet during the larval development of pacu Piaractus mesopotamicus. The statistical results showed significant effect of age and treatment. However, no significant effect was observed for the interaction of both factors. Glycine, lysine and betaine are considered good stimulants of the pacu feeding behavior.
\end{abstract}

Key Words: amino acids, ingestion rates, Piaractus mesopotamicus, stimulants

\section{Introdução}

A quimiorrecepção é um sentido importante para a detecção e o comportamento de procura de alimento em muitos animais aquáticos (Harpaz et al., 1987), pois esses organismos liberam vários compostos na água na osmorregulação, metabolismo e eventual morte (decomposição). Além disso, a água, como solvente universal, disponibiliza esses compostos para vários organismos (Sorensen \& Caprio, 1997). Em peixes, o comportamento alimentar, a resposta de alarme, o reconhecimento de predadores e a comunicação por feromônios iniciam com detecção do odor apropriado (Hara, 1992).

Estudos sobre a estimulação química de peixes e crustáceos podem disponibilizar informações sobre o comportamento quimiossensorial e fisiológico desses animais, bem como gerar informações que podem ser aplicadas na indústria de rações, pois algumas substâncias melhoram a atratividade e a palatabilidade das dietas, aumentando assim o consumo (Coman et al., 1996). Vários estudos indicam que os aminoácidos livres e a betaína são estimuladores da alimentação para peixes (Knutsen, 1992; Harada, 1992; Kolkovski et al., 1997). Lipschitz \& Michel (1999) observaram que juvenis de zebrafish, Danio rerio, podem discriminar, pelo olfato, a L-arginina de análogos estruturais.

No processo de alimentação, a transição da captura de alimento vivo para a de alimento inerte em larvas de peixes é um desafio a ser vencido na larvicultura intensiva de muitas espécies. Kolkovski et al. (2000) consideram que a atratividade da dieta é determinante do sucesso na adaptação de larvas de peixes a dietas inertes.

A criação intensiva de larvas de peixes no Brasil é uma realidade, principalmente para espécies de alto valor comercial, como pintado (Pseudoplatystoma coruscans) e dourado (Salminus brasiliensis). O emprego da larvicultura intensiva na criação de pacus tem sido investigada quanto à sua viabilidade zootécnica e econômica (Jomori et al., 2003; 2005) e alimentação inicial (Macedo-Viegas et al., 2004; Tesser et al., 2005). No entanto, pouco se sabe sobre os efeitos da utilização de estimulantes químicos no comportamento alimentar de larvas de pacu e sobre a 
possibilidade de sua utilização para melhorar a ingestão de dietas inertes. O objetivo neste trabalho foi investigar o efeito estimulante de cinco aminoácidos (alanina, arginina, glicina, histidina e lisina), da betaína e de suas misturas sobre a taxa de ingestão de dieta microencapsulada ao longo do desenvolvimento larval de pacus, Piaractus mesopotamicus.

\section{Material e Métodos}

Larvas de pacu (Piaractus mesopotamicus) recémeclodidas foram estocadas em tanques de $100 \mathrm{~L}$, com renovação de água e aeração constantes, onde foram alimentadas com náuplios de Artemia até 12 horas antes do início do dia experimental, quando a alimentação foi suspensa e as larvas transferidas para as unidades experimentais. Cada unidade experimental foi mantida com 10 larvas e consistiam de recipientes plásticos de $400 \mathrm{~mL}$ de volume, abastecidos com 200 mL de água. Para determinação da taxa de ingestão de dieta microencapsulada, foi utilizada a metodologia adaptada de Dou et al. (2000).

A água utilizada nos tanques de manutenção e nas unidades experimentais era proveniente de poço artesiano. A temperatura foi mantida a $29^{\circ} \mathrm{C}$ por meio de aquecedores submersos e o pH médio, a 8,2. Após 12 horas de permanência das larvas sem alimentação, foi introduzida com uma pipeta automática nas unidades experimentais uma solução-estoque das substâncias-teste, $2 \mathrm{~mL}$, que resultou em concentração final de $1 \times 10^{-4} \mathrm{M}$ para as substâncias individuais (L-alanina, L-arginina, glicina, L-histidina, L-lisina e betaína), de $5 \times 10^{-4}$ para a mistura de todos os aminoácidos e de $6 \times 10^{-4}$ para mistura de todos aminoácidos mais betaína. A concentração individual de cada substância estimulante foi escolhida de acordo com os resultados obtidos com larvas recém-eclodidas de Danio rerio (Lindsay \& Vogt, 2004). Soluções-estoque dos cinco aminoácidos testados, da betaína e das suas misturas foram preparadas com água destilada e preservadas a $4^{\circ} \mathrm{C}$. Novas soluções foram preparadas para cada dia experimental.

Após 5 minutos de exposição das larvas às soluções, uma quantidade considerada em excesso de dieta microencapsulada foi fornecida às larvas duas vezes durante o período de 30 minutos de alimentação (total 2 g). Foi avaliado também um grupo controle, que permaneceu nas mesmas condições previamente descritas, mas que recebeu água destilada para simular as mesmas condições. Após o período de alimentação, as larvas foram rapidamente abatidas com gelo para prevenir que regurgitassem o alimento ingerido. As larvas mortas foram fixadas em formol
10\% tamponado para posterior observação do conteúdo do trato digestório, sob estereoscópio.

As coletas de dados ocorreram no $15^{\circ}, 20^{\circ}$, $25^{\circ}$ e e $30^{\circ}$ dias de vida, correspondendo ao $10^{\circ}$, 15으, 20으 e 25으 dias de alimentação exógena. Em cada dia experimental, foi realizada biometria em aproximadamente dez larvas para a caracterização morfométrica das mesmas (Tabela 1). As larvas foram pesadas individualmente em balança analítica digital.

O experimento foi realizado em delineamento inteiramente casualizado constando de 9 tratamentos em esquema fatorial $9 \times 4$, composto de nove substâncias-teste e quatro idades, avaliados com três repetições de cada tratamento. Aos valores de taxa de ingestão foi aplicada análise de variância paramétrica utilizando-se o Programa SAS, versão 8.0 e (SAS Institute, Inc., EUA). Nos resultados em que houve diferença significativa $(\mathrm{P}<0,05)$, as médias foram contrastadas pelo teste LSD (Least Significant Difference) a $5 \%$ de probabilidade.

Para satisfazer as premissas da análise de variância, os resultados foram testados quanto à normalidade (ShapiroWilk) e à homogeneidade de variâncias (Levene) e previamente transformados (raiz quadrada $\mathrm{X}+0,5$ ) (Sokal \& Rohlf, 1995).

Tabela 1 - Peso (g) e comprimento total de larvas de pacu em diferentes idades alimentadas com dietas suplementadas com aminoácidos

\begin{tabular}{ccc}
\hline Dias de vida & Peso $(\mathrm{g})$ & Comprimento total $(\mathrm{mm})$ \\
\hline 15 & $0,0081 \pm 0,0026$ & $9,76 \pm 0,44$ \\
20 & $0,0152 \pm 0,0028$ & $11,10 \pm 0,73$ \\
25 & $0,0374 \pm 0,0144$ & $12,85 \pm 1,53$ \\
30 & $0,0600 \pm 0,0256$ & $14,60 \pm 2,32$ \\
\hline
\end{tabular}

\section{Resultados e Discussão}

Houve efeito significativo $(\mathrm{P}<0,05)$, tanto da idade como da dieta sobre a taxa de ingestão (Tabela 2). No entanto, não houve significância estatística para a interação dos dois fatores $(\mathrm{P}>0,05)$. $\mathrm{O}$ aumento da ingestão de grânulos de ração por larvas de pacu com o avanço da idade também está relacionado ao desenvolvimento muscular e das nadadeiras, aumentando a capacidade natatória e o desenvolvimento estrutural da cabeça. Esses aumentos no desenvolvimento são necessários para manutenção do rápido crescimento larval (Osse et al., 1997).

Os aminoácidos glicina (aminoácido dispensável) e lisina (aminoácido básico) e a betaína podem ser considerados bons estimuladores do comportamento alimentar de larvas de pacu, pois resultaram em acréscimo 
médio aproximado de 54, 38 e 28\%, respectivamente, em relação ao grupo controle e diferiram estatisticamente do mesmo $(\mathrm{P}<0,05)$. A arginina determinou valores intermediários de taxa de ingestão. Interessante notar que Tesser et al. (2005), estudando os efeitos da suplementação de arginina nas formas livre (L-arginina) e dipeptídica em dieta purificada para juvenis de pacu, não notaram diferenças significativas no consumo instantâneo da dieta, mesmo em comparação ao grupo controle, que não recebeu suplementação. Kolkovski et al. (1997), em pesquisa com larvas de Sparus aurata, observaram que os aminoácidos arginina, alanina, glicina, além da betaína, promoveram efeitos significativos na taxa de ingestão de dietas inertes. Por outro lado, juvenis e adultos de salmão-do-atlântico (Salmo salar) manifestaram respostas comportamentais negativas em relação à presença dos aminoácidos arginina, histidina e lisina.

Coman et al. (1996) relataram que o efeito estimulador do comportamento de natação do camarão Penaeus monodon é maior quando utilizada mistura de vários aminoácidos em comparação ao uso de um único aminoácido. Ainda, Coman et al. (1996) ressaltaram que as interações sinérgicas entre os aminoácidos individuais poderiam produzir uma solução mais atrativa. O fornecimento da mistura de todos os aminoácidos avaliados neste estudo e de todos os aminoácidos mais a betaína não promoveu aumento significativo da taxa de ingestão da dieta pelas larvas de pacu em relação à dieta controle $(\mathrm{P}>0,05)$. Yacoob \& Browman (2007) observaram que a mistura de glicina, prolina, betaína, arginina e alanina não resultou em acréscimo

Tabela 2 - Taxa de ingestão (grânulos/larva em 30 minutos) de alimento entre larvas de pacu alimentadas com dietas suplementadas com aminoácidos

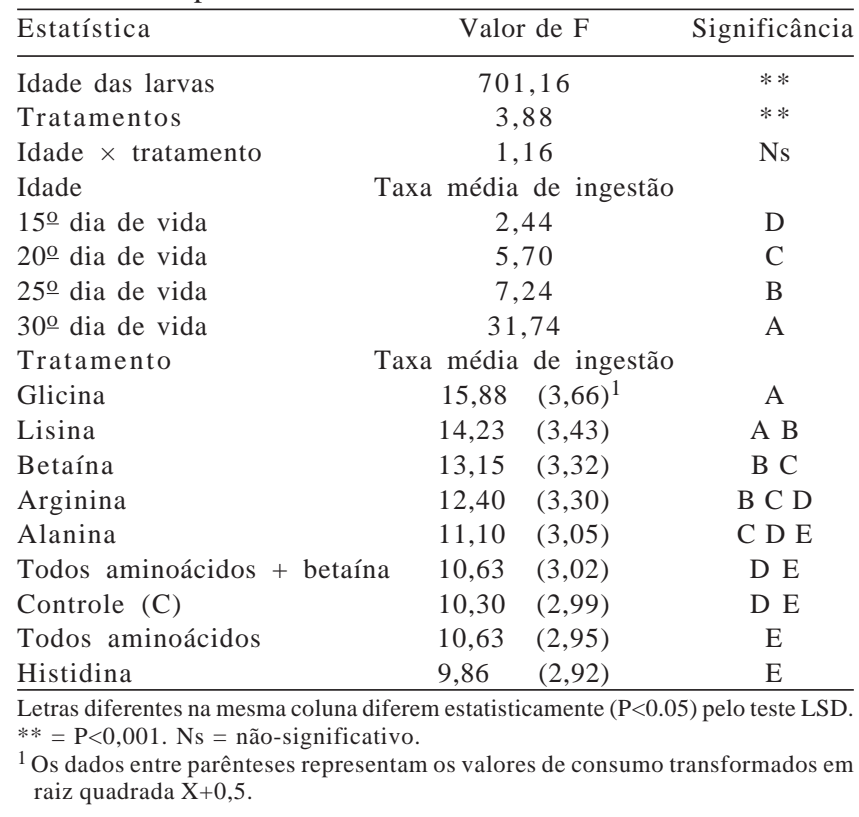

no comportamento de procura alimentar entre juvenis de linguado-do-atlântico (Hippoglossus hippoglossus). Por outro lado, os extratos de camarão e de lula promoveram aumento significativo do comportamento de procura de alimento. De acordo com os autores, os aminoácidos testados são encontrados em grande quantidade em tecidos de camarões e lulas, principais itens da alimentação do linguado-do-atlântico. Velez et al. (2007) avaliaram o efeito atrativo de extratos de poliquetos e de substâncias liberadas por esses organismos em seu metabolismo para o linguado Solea senegalensis e verificaram que a mistura de aminoácidos sintéticos foi muito menos atrativa para essa espécie que as substâncias liberadas pelos poliquetos, o que poderia indicar que outras substâncias atrativas estariam sendo liberadas ou que aminoácidos sintéticos não têm o mesmo efeito que os aminoácidos naturais.

Vários fatores podem explicar essas diferenças entre a atratividade promovida pelos aminoácidos, entre eles, a composição das misturas, a concentração de cada composto, a natureza ecológica e taxonômica de cada espécie, além do número de animais testados. Bórquez \& Cerqueira (1998) observaram que o comportamento alimentar de juvenis de Centropomus undecimalis em resposta à substância estimulante foi maior em experimentos com dois peixes que em estudos com apenas um peixe. Estudos complementares devem ser conduzidos para determinação da influência de misturas de substâncias sobre o comportamento alimentar de larvas de pacu.

Não se espera que a concentração das substâncias testada nesta pesquisa $\left(10^{-4} \mathrm{M}\right)$ explique o fato de algumas substâncias não apresentarem efeito estimulador em larvas de pacu. Shparkovskiy et al. (1983) e Sola et al. (1993) notaram que o salmão e a enguia (Anguilla anguilla), respectivamente, podem detectar a presença de substâncias presentes na água até na concentração de $10^{-9} \mathrm{M}$. No entanto, como alegado por Shparkovskiy et al. (1983), a influência de cada aminoácido sobre o comportamento dos peixes pode variar com a idade dos animais.

Pesquisas recentes comprovam que embriões de pacu com 17 horas pós-fertilização $\left(28^{\circ} \mathrm{C}\right)$ já apresentam a vesícula olfativa diferenciada e, aos 3-4 dias póseclosão, coincidindo com o início da limentação exógena, o epitélio olfativo já está completamente diferenciado, com células ciliadas não-sensoriais e células receptoras com microvilosidades presentes. Segundo Clavijo-Ayala (2008), o sentido olfativo se desenvolve muito rapidamente em larvas de pacu, provendo um sentido vital para sua sobrevivência. Lindsay \& Vogt (2004) citaram que larvas de zebrafish, ao eclodir, parecem possuir o sistema olfatório 
anatomicamente e bioquimicamente funcional. Mesmo que nesta pesquisa não tenha sido avaliada a presença de estruturas quimioreceptoras, considera-se que as larvas já apresentavam o epitélio completamente funcional a partir dos 10 dias de vida, portanto, estavam aptas a detectar as substâncias oferecidas como estimulantes.

O conhecimento de substâncias que possuem efeitos estimulantes para larvas de peixes representa não apenas interesse comercial, pelo seu uso como atrativos em dietas, como determinado para larvas de Perca flavescens (Kolkovski et al., 2000), mas também pela ecologia larval no ambiente natural. As larvas de pacu, ao eclodir, possuem reservas vitelinas que se exaurem muito rapidamente (3 - 5 dias), sendo forçadas à procura por alimento exógeno e ao desenvolvimento de estratégias para evitar predadores ainda com a maioria dos órgãos em desenvolvimento. A quimiorrecepção, realizada por meio do sistema quimiossensorial, é fundamental para a detecção de possíveis presas no ambiente, como já comprovado para espécies marinhas (Døving et al., 1994), e mais recentemente para larvas de pintado Pseudoplatystoma coruscans. Cestarolli (2005) estudou o desenvolvimento das estruturas sensoriais e concluiu que o olfato e o paladar são importantes para o comportamento de alimentação.

\section{Conclusões}

Os aminoácidos glicina e lisina e a betaína são efetivos estimuladores da ingestão alimentar em larvas de pacu e são eficientes como atrativos em dietas para essa espécie.

\section{Agradecimentos}

À Coordenação de Aperfeiçoamento de Pessoal de Nível Superior (CAPES), pela concessão de bolsa de doutorado ao primeiro autor.

\section{Referências}

BÓRQUEZ, A.; CERQUEIRA, V.R. Feeding behavior in juvenile snook, Centropomus undecimalis. I. Individual effect of some chemical substances. Aquaculture, v.169, p.25-35, 1998.

Cestarolli, M.A. Larvicultura do pintado Pseudoplatystoma coruscans (Agassis, 1829): aspectos da alimentação e do desenvolvimento de estruturas sensoriais. 2005. 110f. Tese (Doutorado em Aquicultura) - Centro de Aqüicultura da Unesp, Jabticabal.

CLAVIJO-AYALA, J.A. Ontogenia do Sistema sensorial de pacu Piaractus mesopotamicus (Holmberg, 1887) (Characidae: Serrassalmidae). 2008. 53f. Dissertação (Mestrado em Aquicultura) - Centro de Aqüicultura da Unesp, Jaboticabal.
COMAN, G.J.; SARAC, H.Z.; FIELDER, D. et al. Evaluation of crystalline amino acids, betaine and amp as food attractants of the giant tiger prawn (Penaeus monodon). Comparative Biochemistry and Physiology, v.113, p.247-253, 1996.

DOU, S.; SEIKAI, T.; TSUKAMOTO, K. Feeding behaviour of japanese flounder larvae under laboratory condition. Journal of Fish Biology, v.56, p.654-666, 2000.

DØVING, K.B.; MÅRTØL, M.; ANDERSEN, J.R. et al. Experimental evidence of chemokinesis in newly hatched cod larvae (Gadus morhua L.) Marine Biology, v.120, p.351-358, 1994.

HARA, T.J. Fish chemoreception. Amsterdam: Elsevier Press, 1992. 392p.

HARADA, K. Effect of attractant and repellent mixtures on behaviour of the oriental weatherfish (Misgurnus anguillicaudatus). Nippon Suisan Gakkaishi, v.58, p.1427-1430, 1992.

HARPAZ, S.; KAHAN, D.; GALUN, R. et al. Response of freshwater prawn Macrobrachium rosenbergii to chemical attractants. Journal of Chemical Ecology, v.13, p.1957-1965, 1987.

JOMORI, R.K.; CARNEIRO, D.J.; MALHEIROS, E.B. et al. Growth and survival of pacu Piaractus mesopotamicus (Holmberg, 1887) juveniles reared in ponds or at different initial larviculture periods indoors. Aquaculture, v.221, p.277-287, 2003.

JOMORI, R.K.; CARNEIRO, D.J.; MARTINS, M.I.E.G. et al. Economic evaluation of Piaractus mesopotamicus juvenile production in different rearing systems. Aquaculture, v.243, p.175-183, 2005.

KOLKOVSKI, S.; ARIELI, A.; TANDLER, A. Visual and chemical cues stimulate microdiet ingestion in sea bream larvae. Aquculture International, v.5, p.527-536, 1997.

KOLKOVSKI, S.; CZESNY, S.; DABROWSKI, K. Use of krill hydrolysate as a feed attractant for fish larvae and juvenile. Journal of the World Aquaculture Society, v.31, p.81-88, 2000.

KNUTSEN, J.A. Feeding behavior of North Sea turbot (Scophthalmus maximus) and Dover sole (Solea solea) larvae elicted by chemical stimuli. Marine Biology, v.113, p.543-548, 1992.

LINDSAY, S.M.; VOGT, R.G. Behavioral responses of newly hatched zebrafish (Danio rerio) to amino acid chemostimulants. Chemical Senses, v.29, p.93-100, 2004.

LIPSCHITZ, D.L.; MICHEL, W.C. Physiological evidence for the discrimination of L-arginine from structural analogues by the zebrafish olfactory system. Journal of Neurophysiology, v.82, p.3160-3167, 1999.

MACEDO-VIEGAS, E.M.; PORTELLA, M.C.; CARNEIRO D.J. Utilization of fish protein hydrolysate in prepared diets for pacu, Piaractus mesopotamicus, larvae. Journal of Applied Aquaculture, v.14, p.101-112, 2004.

OSSE, J.W.M.; VAN DER BOOGAART, J.G.M.; VAN SNIK, G.M.J. et al. Priorities during early growth of fish. Aquaculture, v.155, p.249-258, 1997.

SHPARKOVSKIY, I.A.; PAVLOV, I.D.; CHINARINA, A.D. Behavior of young hatchery-reared Atlantic Salmo salar, (Salmonidae) influenced by amino acids. Journal of Ichthyology, v.23, p.140-147, 1983.

SOKAL, R.R.; ROHLF, F.J. Biometry. The principles and practice of statistics in biological research. 3.ed. New York: W.H. Freeman Publisher, 1995. 886p.

SOLA, C.; SPAMPANATO, A.; TOSI, L. Behavioural responses of glass eels (Anguilla anguilla) towards amino acids. Journal of Fish Biology, v.42, p.683-691, 1993.

SORENSEN, P.W.; CAPRIO, J. Chemoreception. In: EVANS, D.H. (Ed.). The physiology of fishes. Boca Raton: CRC Press, 1997. p.375-405.

TESSER, M.B.; CARNEIRO, D.J.; PORTELLA, M.C. Co-feeding of pacu (Piaractus mesopotamicus, Holmberg, 1887) larvae with Artemia and microencapsulated diet. Journal of Applied Aquaculture, v.17, p.47-59, 2005.

TESSER, M.B.; TERJESEN, B.F.; ZHANG, Y. et al. Free- and peptide- based arginine dietary supplementation for the South American fish pacu (Piaractus mesopotamicus). Aquaculture Nutrition, v.11, p.443-453, 2005. 
VELEZ, Z.; HUBBARD, P.C.; HARDEGE, J.D. et al. The contribution of amino acids to the odour of a prey species in the Senegalese sole (Solea senegalensis). Aquaculture, v.265, p.336-342, 2007.
YACOOB, S.Y.; BROWMAN, H.I. Prey extracts evoke swimming behavior in juvenile Atlantic halibut (Hippoglossus hippoglossus). Aquaculture, v.270, p.570-573, 2007. 